\title{
CALIDAD DE LA EDUCACIÓN SUPERIOR: UN ANÁLISIS DE SUS PRINCIPALES DETERMINANTES*
}

\author{
Diana Lago de Vergara ${ }^{1}$ \\ Audin Aloiso Gamoba Suárez ${ }^{2}$ \\ Alexander Javier Montes Miranda ${ }^{3}$
}

\begin{abstract}
RESUMEN
El presente artículo presenta una revisión de la literatura de la investigación titulada calidad de la educación superior: imaginarios institucionales y de actores educativos. El texto muestra un análisis sobre los principales determinantes de la calidad de la educación superior en temas relacionados con la globalización, el tratado de Bolonia y su impacto en Europa y América Latina, así como los discursos institucionales sobre la calidad y categorías como investigación, docencia y gestión como factor decisivo en los planes de mejora de las instituciones.
\end{abstract}

\section{PALABRAS CLAVE}

Actores educativos, calidad, docencia, educación superior, gestión, investigación

\begin{abstract}
This article presents a literature review of research entitled quality of higher education: institutional and educational imaginary actors. The text shows an analysis of the main determinants of the quality of higher education on issues related to globalization, the treaty of Bologna and its impact on Europe and Latin America, as well as institutional discourses on quality category as research, teaching and management as instrumental in the improvement plans of institutions factor
\end{abstract}

\section{KEY WORDS}

Educational stakeholders, quality teaching, higher education, management, research.

Depositado en febrero 16 de 2014, aprobado en mayo 23 de 2014.

* Proyecto avalado por el grupo de investigación en estudios sociales y pedagogía para la paz (GIESPPAZ), categoría C (COLCIENCIAS) de la Universidad Francisco de Paula Santander (Cúcuta).

1 Doctora en Filosofía y Ciencias de la Educación de la Universidad Complutense de Madrid (España), Magister en Proyectos de Desarrollo Social de la Universidad del Norte (Colombia). Grupo de investigación RUECA. Docente investigadora de la Universidad de Cartagena. Correo electrónico: dianalago20@yahoo.es

2 Magister en Pedagogía de la Universidad Industrial de Santander, Doctorando en Ciencias de la Educación (RUDECOLOMBIA). Grupo de investigación RUECA. Docente Investigador de la Universidad Francisco de Paula Santander - Cúcuta. Correo electrónico: docaudin@ gmail.com

3 Magister en Educación, Doctorando en Ciencias de la Educación (RUDECOLOMBIA - Cade-Universidad de Cartagena). Grupo de investigación RUECA. Docente de la Universidad de Córdoba - Colombia. Correo electrónico: amontes20@gmail.com 


\section{INTRODUCCIÓN}

El término calidad, está presente en todas las acciones y escenarios de la vida cotidiana. La calidad se relaciona con eficacia, eficiencia, un estado de satisfacción, conformidad y servicio. La calidad es un concepto complejo de definir y tiene distintos significados para todos los sujetos. Por ejemplo, la calidad según Díaz (2008), consiste en satisfacer los estándares establecidos para los insumos, procesos y, principalmente, resultados. Así mismo, uno de los conceptos con los que más se relaciona la calidad es con la excelencia, "la cual supone el compromiso de todos los integrantes de una organización para lograr un producto lo mejor posible, empleando los mejores componentes, la mejor gestión y los mejores procesos" (Miranda, Chamorro \& Rubio, 2007, p. 8).

Para Lago, López, Municio, Ospina y Vergara (2013)

La calidad es el resultado de comparar las expectativas con la percepción del servicio recibido. Esto significa que la medida de la calidad está en la satisfacción de las personas y en el valor de lo que reciben. La efectividad, como relación entre las necesidades sentidas y los resultados percibidos es la única escala válida para medir la calidad. El éxito de los programas o de las organizaciones radica esencialmente en responder a la maximización de esta proporción ( $\mathrm{p}$. 37)

Es importante señalar que el campo conceptual de la calidad está definido por dos posturas importantes: la calidad desde lo objetivo y la calidad desde lo subjetivo. El primero según Municio (2000), se relaciona con lo cuantificable, verificable con algún estándar, descriptor o indicador previamente determinado, y desde lo subjetivo, el mismo autor señala que la calidad se refiere al valor que le dan las personas a lo que responde a sus deseos, a su deber ser y a la comparación de sus expectativas con su propia percepción de la realidad. En este sentido, la calidad se convierte en una determinación del ser, que se refiere no solo a la materia sino principalmente al ser mismo de las cosas, a sus caracteres psicológicos y espirituales (García, 1991, p. 9).

Por otra parte, la calidad desde el contexto educativo se puede definir como "el modo de ser de la educación que reúne las características de integralidad, coherencia y eficacia" (García, 1991, p. 10). Así mismo, el Consejo Nacional de Acreditación - CNA (2010), afirma que la calidad de la educación es un atributo de servicio público de la educación en general, y en particular, al modo como este servicio se presta, según el tipo de institución de que se trate. Igualmente, alude y supone "el esfuerzo continuo de las instituciones por cumplir de forma responsable con las exigencias propias de cada una de sus funciones como son: la investigación, la docencia y la proyección social" (p. 11).

De otro modo, es importante señalar que gran parte de los estudios que se han realizado sobre la calidad de la educación superior toman fuerza posterior al tratado de Bolonia de 1999 donde se muestran los impactos de este acuerdo tanto en Europa (García, 2011; EURYDICE, 2009 \& Díaz, 2013) como en Latinoamérica (Olvera, 2008) y Gacel, 2011) y donde se resaltan los grandes aportes y el importante referente que ha sido, para los procesos de mejora de la educación superior.

Cuando se habla de investigaciones sobre calidad de la Educación Superior se puede afirmar que la gran mayoría de estudios se centran en medir los impactos de los procesos de autoevaluación y acreditación de las IES (Scharager \& Aravena, 2010 y Navarro, 2007), comparar los diferentes sistemas de aseguramiento de la calidad (Torres \& Zapata, 2012 y Lemaitre, 2004 ) y analizar los modelos de evaluación de la calidad de los programas académicos (González, 2005).

\section{EL TRATADO DE BOLONIA COMO RUTA HACIA LA CALIDAD DE LA EDU- CACIÓN SUPERIOR}

Uno de los referentes más importantes para la investigación en calidad de la Educación Superior es el tratado de Bolonia, constituido en 
1999, este tratado revolucionó la educación superior en Europa con importantes efectos en América Latina y el Caribe. La ruta de navegación propuesta por Bolonia buscaba crear un Espacio Europeo de Educación Superior competitivo, atractivo tanto para los estudiantes como para los docentes, y atractivo para otros países. Algunos de los principales cambios que se pretendieron en este tratado fueron las adaptaciones curriculares y las adaptaciones tecnológicas, la adopción de un sistema de titulaciones universitarias fácilmente comparable en toda Europa lo que daba apertura a la movilidad de estudiantes y profesores investigadores por las universidades europeas, a la vez que se fomentaba el aprendizaje continuo y la calidad de la Educación (Ceballos, Cantarero \& Pascual, 2004).

Desde la puesta en marcha de este tratado han sido diversas las investigaciones que han surgido en los diferentes ámbitos de la calidad de la Educación explorando su impacto en Europa y América Latina.

En Europa, particularmente EURYDICE (2009), presenta un informe sobre los principales avances del tratado de Bolonia en aspectos relacionados con la estructura del Grado-Máster, el sistema europeo de transferencia y acumulación de créditos, el suplemento europeo al título, los marcos nacionales de cualificación y la movilidad y portabilidad de la ayuda económica a los estudiantes. El informe muestra, entre otros aspectos, que a través de la implantación de importantes reformas se ha logrado una mayor compatibilidad entre las distintas estructuras de la oferta de programas de educación superior en los países europeos.

Otros estudios importantes realizados desde la mirada europea, es la evaluación de la calidad universitaria por parte de los estudiantes (González, 2005), donde los determinantes principales apuntan a obtener una capacidad de reflexión, un autoaprendizaje y la formación, para la consecución del empleo. En esta misma dimensión desde la perspectiva de los estudiantes ante los cambios con la llegada de Bolonia (Galán, 2009), se analiza la garantía de calidad y la implicación que tiene en ella el estudiante, dentro de un proceso de cambio de su figura en el sistema universitario español. La investigación revela que hay una clarificación de la perspectiva estudiantil, la inclusión de nuevos temas y cuestiones y la sugerencia de nuevos proyectos de evaluación; en la autoevaluación ha ampliado la presencia y el compromiso estudiantil, ha producido informes estudiantiles y ha permitido un liderazgo para el desarrollo local de la calidad después de la evaluación. Así mismo, se evidencian los beneficios constatados que la participación estudiantil ha aportado en la garantía de calidad en el resto de Europa. Aunque los cambios que trajo Bolonia no solamente son de participación de los estudiantes en su formación sino también, de una nueva mirada a las formas de aprendizaje (Díaz, 2010; Díaz, Maroto, Barcala, \& López 2012), y a las competencias que deben desarrollar los estudiantes (Montero, 2010; Pales, Nolla, Oriol, \& Gual, 2010 y Rodríguez \& Vieira, 2009)

Como complemento a la mirada de la calidad de la educación en Europa es importante analizar cómo los sistemas de indicadores Europeos suponen un constructo teórico y metodológico sobre la calidad de la educación superior. La investigación de Chacón y Pérez (2001), devela de cierta manera la importancia y el carácter mediador que tienen los indicadores entre lo teórico y lo empírico, entre los constructos teóricos y el mundo externo. De igual forma, estos autores concluyen que disponer de información de calidad sobre distintas dimensiones de interés en el ámbito universitario, se convierte en un recurso imprescindible para plantear un acercamiento a esa meta de difícil concreción como es el aseguramiento de la calidad.

El tratado de Bolonia ha tenido un impacto directo sobre la calidad de la Educación Superior en Europa, al igual que en América Latina y el Caribe. También, los Gobiernos y Universidades de América Latina han iniciado reformas educativas con el fin de:

Homologar las estructuras de las diferentes carreras profesionales y el sistema de evaluación que las acredita para que los profesionales puedan ejercer el proceso de movilidad y todas sus implicaciones en el rubro de la calidad, 
primeramente entre las universidades de su país de origen, posteriormente en toda la población latinoamericana, y así contribuir al acercamiento regional y global, de tal forma que se pueda hablar de un proyecto transcontinental de tal magnitud como el de la UE y ALC (Bulgarín, 2008, p. 57).

Investigaciones como la de Gacel (2011), han podido corroborar que el impacto de Bolonia en América Latina ha sido un modelo a seguir, puesto que este fue promulgado frente a unas estructuras académicas con un alto grado de innovación, enriqueciendo el debate sobre la modernización educativa de América Latina.

Bruner (2008), habla de un notable avance en la educación superior Latinoamericana favorecido por lo que él llama "Ecos de Bolonia" y estos sugieren "nuevas conversaciones, propone tópicos de análisis, muestra posibles soluciones y enseña políticas y procedimientos para alcanzarlas" (p.138).

Aunque no solo en las reflexiones sobre nuevas políticas ha influenciado Bolonia, elementos muy importantes como la necesidad de formación docente, movilidad de estudiantes y docentes (Cordera \& Santamaría, 2008), y un tema de gran relevancia como las competencias (Aboites, 2010) tanto en docentes como en estudiantes han sido aprendizajes de Bolonia.

Es fundamental reconocer que el tratado de Bolonia es un antecedente de gran relevancia en la política que configura el sistema de aseguramiento de la calidad tanto en Europa como en América Latina, y que este plan, en un principio utópico, ha impactado de manera positiva en la calidad de la educación en gran parte del mundo.

\section{LA CALIDAD DE LA EDUCACIÓN SU- PERIOR: LAS VOCES DE LOS ACTORES EDUCATIVOS}

\section{Las voces de los estudiantes}

Uno de los principales actores que intervienen en los procesos formativos de la educación superior son los estudiantes, por lo que es impor- tante conocer las representaciones que tienen estos actores sobre su modo de ver la educación superior y su visión sobre la calidad de la misma.

En este sentido, se puede evidenciar que la función docente es una de los principales determinantes de calidad para los estudiantes y, de esta manera, "un profesor de calidad" es aquel que según sus estudiantes, evalúa con justicia, organiza sus clases, es respetuoso (Casero, 2010) y se expresa con claridad (Apodaca \& Grad, 2002). Estas aseveraciones concuerdan con los estudios de Cabalín y Navarro (2008) quienes señalan que un buen profesor es el que presenta atributos de valor como el de ser respetuoso, responsable, comprensivo, empático, puntual, inteligente y amable, lo cual según estos autores, fortalece las relaciones interpersonales y promueve el aprendizaje desde un punto de vista afectivo.

No obstante, de las opiniones positivas de los estudiantes sobre sus profesores, hay algunas que se contradicen y ven al profesor como una persona que maltrata y acosa al estudiante (Iglesias, Saldívar, Bermúdez \& Guzmán, 2005). Otros estudios realizan importantes apreciaciones sobre lo que según los estudiantes deben mejorar los profesores, referidos a aspectos como "la gestión, el contexto de valor, la creatividad expositiva, la utilización de recursos y el estímulo en la participación de los alumnos" (Mayor, 1997, p. 21).

En conclusión, la valoración realizada por los estudiantes sobre la calidad de los profesores, está ligada como lo señala Leiva (2011), a "las estrategias metodológicas y evaluativas, sobre todo con los principios, valores, convicciones y traducción práctica de los mismos, desde la perspectiva de la coherencia entre la teoría y práctica del docente y entre lo que dice y hace" (p. 175).

Otra categoría importante de analizar desde la perspectiva de los estudiantes, es la que hace relación a la gestión universitaria, la cual garantiza sostenibilidad, bienestar y buen servicio. En este sentido Torres \& Araya (2010), construyeron una escala para medir la calidad del 
servicio de las universidades chilenas, este estudio buscó construir una escala con suficiente grado de fiabilidad, validez y dimensionalidad, que permitiera medir la calidad del servicio de las universidades desde la perspectiva de los estudiantes. El trabajo concluye que la percepción general y particular de las universidades en la mente de los estudiantes y en la opinión pública es relevante para la escogencia de universidades para cursar la carrera profesional, ya que la decisión se encuentra influenciada por su entorno cercano y grupo de referencia.

Otros estudios relacionados con la gestión, muestran como determinante de la calidad los espacios físicos adecuados, un ambiente institucional donde se den relaciones humanas gratas, convivencia enriquecedora, calidad del servicio y buena calidad de vida, y donde se tenga oferta de opciones recreativas y disponibilidad de tiempo para el esparcimiento (Demicheli, 2009).

\section{Las voces de los profesores}

Al igual que los estudiantes, los educadores son actores principales en la educación superior y su opinión sobre la calidad es igual de valiosa. En este sentido, numerosos estudios han develado diferentes representaciones y opiniones que tienen los educadores al respecto.

Por ejemplo, en el contexto europeo, Cardona, Ayesta, Mijangos y Olaskoaga (2009), realizaron una investigación donde buscaron identificar la opinión de los docentes sobre la calidad de la educación superior. Los resultados más relevantes de este estudio cuantitativo muestran que las categorías de opinión de mayor frecuencia entre los profesores hacen referencia al desarrollo de las capacidades del estudiante para avanzar en su propia transformación, satisfacer las expectativas de todos los involucrados (alumnos, profesores, instituciones y sociedad) $\mathrm{y}$ formar estudiantes capaces de asumir un compromiso social. Lo que evidencia que las opiniones sobre la calidad de la educación desde la mirada de los docentes se encuentran supeditada y directamente relacionada con la función docente (Arbeláez, 2004), lo cual se traduce en calidad de la enseñanza y calidad del aprendiza- je como lo menciona Biggs (2006), en su texto calidad del aprendizaje universitario.

Por su parte, Correira y Miranda (2012), señalan que no solamente la calidad de la educación superior se mide exclusivamente por el proceso de enseñanza - aprendizaje, si no que existen otros factores que intervienen en esta valoración como: la gestión universitaria que se manifiesta en el servicio prestado por las bibliotecas universitarias, los servicios de acción social, los servicios académicos, estado de los salones, edificios y espacios recreativos (Murcia, 2009). Es importante señalar que estas formas de representación de la calidad según los profesores, surge desde su misma experiencia, lo que permite valorar la calidad de forma fundamental desde tres campos: docencia, gestión e investigación (Lafuente, 2010).

\section{LA CALIDAD DE LA EDUCACIÓN SUPE- RIOR: UNA MIRADA DESDE LOS DIS- CURSOS INSTITUCIONALES}

Tal vez uno de los textos más críticos sobre la calidad de la educación como discurso institucional es La universidad de papel, de Porter (2005). En este libro, el autor realiza un análisis importante de lo que es la función de la universidad más que a sus políticas que no trascienden las dinámicas de mejora de las instituciones de educación superior mexicanas. El autor señala con gran vehemencia que la calidad de la educación superior son intentos oficiales por elevar la calidad de la universidad, generalmente expresados en la escala "macro" de los documentos normativos y las políticas globales, lo cual solamente produce una universidad en el papel, en la medida de que los documentos que produce no logran descender y mejorar (aunque si alterar negativamente) los ámbitos de la acción diaria, rutinaria. Esta transformación tan (mal) buscada por las autoridades depende de la sustitución de la pasividad o el "egoísmo" que hoy recluye al talento académico en espacios aislados, por un verdadero "individualismo" que permita compartir experiencias y capacidades, no solo con los estudiantes, como cotidianamente ocurre, sino con el resto de los actores de la universidad (p. 151). 
Este texto invita a reflexionar sobre la importancia de generar espacios de discusión sobre la necesidad de abordar la calidad de la educación superior como un trabajo de construcción colectiva donde todos los actores y las políticas institucionales transformen la institución en algo funcional, puesto que es de suponer que las instituciones deben ser funcionales pero no cumplen con el papel de reflexionar como lo señala Castoriadis (2007), sobre ¿cuáles son las necesidades reales de una sociedad? y ¿cómo puede contribuir la institución de educación superior a que la sociedad supere estas necesidades?

Una de las investigaciones más importante que analiza los discursos de calidad en las universidades de una manera crítica es la denominada "Universidad productora de productores entre biopolítica y subjetividad" (Martínez, 2010). El autor realiza un análisis importante donde visibiliza el funcionamiento de los discursos gubernamentales como dispositivos de producción biopolítica de la subjetividad en la educación superior en Colombia, en el período comprendido entre 1991-2005. El aporte de esta investigación radica que en gran parte del texto se analizan los dispositivos discursivos de calidad presentes en las leyes de educación superior en Colombia y hace una interesante reflexión con una mirada crítica al papel de la universidad en la sociedad capitalista y de consumo. Una de las conclusiones que se rescatan de este trabajo es que la "educación superior colombiana particularmente termina subordinando las posibilidades ontológicas de creación a las exigencias innovadoras y empresariales del capital global” (p. 163)

Otros elementos que indudablemente hace parte de la calidad de la Educación superior son las políticas educativas y las reformas a las mismas, estos intentos de los estados por consolidar políticas públicas en pro de la mejora de la calidad de la educación superior también han sido objeto de estudio desde sus mismos discursos. En chile por ejemplo, diversos trabajos han analizado e interpretado las propuestas curriculares que surgen de las políticas públicas que norman y orientan la reforma educacional chilena (Soto, 2002), así mismo se ha explorado desde el análisis de contenido la evolución de las políticas de educación superior y las transformaciones de la educación chilena (Salazar \& Leihy, 2013) y el elemento primordial en la calidad de la educación y su financiamiento (Ponce, 2012).

En el ámbito Latinoamericano, también se han realizado investigaciones donde se analizan los discursos de calidad en las políticas de educación superior con resultados importante que ayudan a develar los discursos presentes en dichas políticas. Estos estudios dan cuenta, por ejemplo, de la globalización y sus efectos en las políticas educativas de educación superior (Bruner, 2010). Otros trabajos abordan el tema de la gestión del conocimiento partiendo de la conformación de redes académicas como determinantes de la calidad, y estudios donde se analizan los debates de las leyes de educación en Latinoamérica principalmente referidos a la formación de docentes y la investigación (Rodríguez, 2006).

En Colombia, de igual manera se han realizado trabajos sobre el análisis de la calidad de la educación presente en los discursos gubernamentales e institucionales. Estos discursos develan las formas y categorías importantes en cuanto a la calidad de la educación, como la financiación (Ruiz, Cano, \& Montes, 2008). Dicho estudio, analizó tres casos en particular: Bogotá, Cali y Medellín durante el periodo 1990 - 2005, y revela que "faltan opciones de financiamiento a largo plazo y un sistema de crédito educativo con diferentes modalidades que se adapten a las demandas de los estudiantes y sus familias" (p. 22). Evidenciando, sin duda alguna, que la financiación es un elemento fundamental en la calidad de la educación superior, particularmente para favorecer la formación y culminación de los estudios, referido al financiamiento y condonación de créditos (Gutiérrez, 2012).

\section{INVESTIGACIÓN Y CALIDAD DE LA EDUCACIÓN}

La investigación junto con la docencia y la extensión hacen parte de los pilares fundamentales de la constitución de la Universidad, no se puede 
concebir una institución de educación superior sin la gestión de estos tres procesos que dinamizan la construcción del conocimiento y el cambio social.

Respecto a la investigación, en Colombia el sistema de aseguramiento de la calidad exige a las IES como política gubernamental que para la apertura de programas, renovación de registros calificados, acreditación institucional y de alta calidad, se debe contemplar la investigación como un elemento necesario en sus estructuras curriculares. En el decreto 1295 de abril de 2010, en el numeral 5.5 , aparece este requerimiento informando a las instituciones de educación superior que promuevan la investigación en sus estudiantes y generen procesos de investigación atendiendo la dinámica que establece COLCIENCIAS ${ }^{3}$ para los grupos de investigación y la producción científica de los docentes. Frente a este requerimiento, se puede apreciar que una limitante para que las instituciones de educación superior sean de naturaleza investigativa son los escasos recursos económicos con los que cuentan para desarrollar estos procesos.

Por su parte, el CNA (2013), concibe la investigación como factor de calidad en las IES, si se satisfacen los aspectos relacionados con la formación investigativa de los estudiantes a nivel de pregrado, dedicación de los docentes a la investigación, producción científica de los profesores, el reconocimiento de los grupos de investigación por parte de COLCIENCIAS u otra institución, y unas políticas claras por parte de las instituciones de educación superior para la financiación de la investigación.

Desde otra mirada, fuera de lo normativo, es innegable que la investigación es un determinante de la calidad de la educación superior y, que además de generar conocimiento, "ésta debe suplir las necesidades profesionales y ocupacionales del entorno laboral con el cual se mantiene estrecha relación" (Lago et al., p. 16).

El proceso investigativo hace presencia y se desarrolla de acuerdo a la naturaleza de la misma institución, por ejemplo, algunas instituciones de educación superior toman como objetivo principal la formación y no ven la investigación como un elemento potente para la transformación y mejora de la calidad del servicio. En este sentido, se deben asumir repercusiones importantes en diversos aspectos, uno de estos es la calidad y transformación de las prácticas pedagógicas del docente, pues el ejercicio investigativo concede al profesor mayor profundidad de conocimientos, permite valorar el papel que desempeña la investigación en el desarrollo de su vida, lo ayuda a mejorar sus métodos ${ }^{4} \mathrm{y}$ resolver problemas educacionales. La investigación abre un nuevo e interesante mundo en el cual se puede experimentar un continuo mejoramiento personal y profesional y esto es una garantía de calidad para la institución. Sin embargo, "el hecho de que un profesor universitario se destaque en la actividad investigadora no implica necesariamente que posea las habilidades para una buena docencia" (Parra, 2004, p. 61). No obstante, el hecho de que el profesor no haga investigación tampoco lo hace un mal docente, solo que se impide conocer múltiples herramientas para la función educadora y para la optimización del aprendizaje de los estudiantes.

\section{DOCENCIA UNIVERSITARIA Y CALI- DAD DE LA EDUCACIÓN}

Al igual que la investigación, la docencia también hace parte fundamental de la educación superior, y ésta infiere directamente en la calidad del servicio que presta la universidad. La calidad de la docencia universitaria "implica un elevado conocimiento sobre cómo funcionan los procesos y las dinámicas de aprendizaje de los sujetos" (Zabalza \& Zabalza, 2010, p.58), el docente no es solo una figura o un actor más en las dinámicas universitarias, es uno de los principales protagonistas en la formación de los estudiantes y en la construcción de conocimiento desde la investigación.

3 Sigla que denomina al Departamento Administrativo de Ciencia, Tecnología e innovación

4 En cuanto a una mejora en la enseñanza de los saberes, o volver enseñable un saber desde la práctica del docente, es importante citar a Parra (2004), quien señala que “...la investigación didáctica es connatural al quehacer universitario, la cual se entiende aquí como aquella cuyo objeto son los procesos de formación, específicamente de enseñanza aprendizaje, y los demás procesos curriculares propios de la función docente"(p. 62) 
Son múltiples las investigaciones que se han realizado sobre la calidad de la docencia en la educación superior, estos estudios dan cuenta entre otros elementos, sobre los estilos de enseñanza (Laudadio, 2012) modelos de evaluación docente (Manzi, 2011; Tejedor \& García, 2010 y Rueda, 2009) y un tema fundamental, las competencias de los profesores de educación superior (Bozu \& Herrera, 2009; Arrufat, Sánchez, \& Santiuste, 2010; Ibarra \& Uribe, 2011 y Tirado, 2009). En este sentido, son variadas las miradas desde la investigación que se le pueden hacer a un buen profesor: actitudes, formas de evaluar, preparación intelectual, formas de enseñar, actividades de investigación, reflexivos de su propia práctica, prudentes y conjugar la exigencia con la comprensión (Mañú, 2011).

\section{Competencias del profesor universitario: $G a-$ rantía de calidad en la docencia}

Las competencias no se pueden centrar solamente en una función laboral, ni en la apropiación del conocimiento de acuerdo a su área disciplinar como lo señala Esteban y Menjívar, (2011), sino que abarca un espectro más complejo que según los autores ocupa todo un conjunto de capacidades que se desarrollan a través de procesos que conducen a la persona responsable a ser competente para realizar múltiples acciones sociales, cognitivas, culturales, afectivas, laborales y productivas.

Marchesi (2007), es uno de los autores que hace referencia a las competencias profesionales docentes y las ha analizado de manera profunda. Este autor señala que el docente debe desarrollar las siguientes competencias para ser competente en su función dentro de la universidad:

Ser competente para favorecer el deseo de saber de los alumnos y para ampliar sus conocimientos, comprometer a los alumnos en sus aprendizajes, ser capaz de responder a la diversidad de los alumnos, estar preparado para incorporar la lectura en la actividad educadora y ... educar en valores y para la ciudadanía. (p. 45)
Otros autores argumentan que existen dos tipos de competencias que debe desarrollar el profesor universitario: las competencias individuales y las competencias colectivas. La primera en la dimensión del saber actuar hace referencia a resolver situaciones en el ámbito pedagógico, a tener dominio propio y de los conocimientos, una comunicación efectiva con todos los actores educativos y la autogestión personal y profesional. La segunda, se manifiesta cuando el docente consigue acuerdos sobre un problema, meta a alcanzar, o situación a mejorar, logra un mismo entendimiento sobre determinada información, elige los modos de cooperación apropiados para las diversas formas de organización y aprende de las experiencias (Jabif, 2007).

\section{GESTIÓN Y CALIDAD DE LA EDUCA- CIÓN SUPERIOR}

La gestión es otro elemento fundamental en la dinámica interna y externa de las instituciones de educación superior. La gestión a modo general está definida como un conjunto de operaciones que se realizan para dirigir y administrar un negocio o una empresa: una buena gestión hace que las empresas funcionen.

En el ámbito educativo, la gestión según Lavín y del Solar (2000), corresponde a toda actividad relacionada con los elementos administrativos y financiaros de una institución, así como a la forma en la cual los directivos, docentes y los estudiantes se organizan. Del mismo modo se refiere a la fijación de calendarios, fijación de horarios, distribución de espacios y otros elementos organizativos. De igual manera, se configura como el conjunto de acciones, relacionadas entre sí, que emprende el equipo directivo de una institución para promover y posibilitar la consecución de la intencionalidad en la comunidad educativa y con ella. Estos autores también plantean que la gestión educativa o gestión escolar posee básicamente seis dimensiones que se mencionan a continuación:

(1) Dimensión pedagógica curricular, corresponde a los fines específicos o razón de ser de la institución en la sociedad. (2) Dimensión administrativa financiera, es la distribución del tiempo, el espacio, el recurso humano y 
los aspectos financieros de la institución. (3) Dimensión organizativa operacional, se refiere a la organización de los subsistemas de la institución; es decir, organización del cuerpo docente y directivos, departamentos administrativos, departamentos académicos, comisiones, entre otros. (4) Dimensión comunitaria, aborda la relación de la institución con el sector externo (sociedad, empresa, organizaciones culturales). (5) Dimensión de convivencia, garantiza el clima organizacional entre los diferentes actores educativos (docentes, administrativos, estudiantes, directivos) y establece las normas internas del actuar en la institución. (6) Dimensión sistémica, es la relación que establece la institución con otras instituciones del sistema educativo (otras universidades, secretarías de educación, etc.).

Uno de los autores que más se ha referido a la gestión como factor de calidad en la educación superior es Municio (2010), quien plantea un enfoque fundamental al respecto: la gestión de la educación por procesos. Este concepto se incrusta en la gestión de la calidad educativa como un nuevo modelo para:

hacer frente a las demandas de la sociedad actual. Por una parte, se aporta tecnología para lograr organizaciones más eficientes $y$, por otra, la orientación a las personas implicadas como razón esencial de la organización y de los procesos. La confluencia se traduce en procesos de mejora continua y el resultado en el valor agregado por la acción del proceso. (p. 113).

\section{CONCLUSIONES}

La revisión de la literatura de este amplio y complejo campo deja elementos importantes para concluir. En primer lugar, es importante conferirle el inegable valor que ha tenido el tratado de Bolonia en los cambios en las instituciones de educacion superior en Europa y su impacto en Ameria Latina. Este tratado y sus condiciones de exigencia han hecho que se transformen los diferentes pilares de la educación como la formación docente, la investigación y la movilidad de estudiantes y profesores en el ambito mundial, entre otros aspectos ya abordados en el escrito. Del mismo modo, se han podido estructurar agencias que fortalecen el aseguramiento de la calidad como el caso de Agencia Nacional de Evaluación de la Calidad y la Acrediatación (ANECA), que con su apoyo y seguimento a las instituciones de Educación Superior han elevalo los indicadores de calidad de las Universidades Europeas.

Desde la visión positiva de la globalización de la educación, se observa que este fenómeno ha permitido que las instituciones de educación superior, tanto en Europa como América Latina, construyan una cultura de la autoevaluación y la calidad, desde sus diferentes procesos académicos $\mathrm{y}$ administrativos. Las instituciones educativas en América Latina, en particular, han sentido el fenómeno de la globalización y están optimizando esfuerzos para hacer que las instituciones guiadas por un sistema de aseguramiento de la calidad más global logren avanzar en importantes temas como los marcos comunes de la educación superior y temas como la formación docente y la consolidacion de convenios funcionales de movilidad internacional.

Por otra parte, es importante destacar que es escasa la literatura sobre investigaciones o experiencias donde se aborde el tema de los actores educativos y sus voces en las transformaciones estructurales de las instituciones de educación superior, salvo la literatura sobre los movimientos sociales estudiantiles y del profesorado. Gran parte de los estudios describen lo que los actores sienten y piensan sobre la calidad educativa, pero no se hace un análisis profundo sobre las tensiones que hay entre estas representaciones y los elementos normativos y estructurales de las instituciones, y cómo estas tensiones han generado cambios importantes.

\section{REFERENCIAS}

1. Aboites, H. (2010). La Educación Superior Latinoamericana y el proceso de Bolonia: de la comercialización al proyecto Tuning de competencias. Revista Cultura y representaciones sociales. 5(9), $122-144$. 


\section{CALIDAD DE LA EDUCACIÓN SUPERIOR: UN ANÁLISIS DE SUS PRINCIPALES DETERMINANTES}

2. Apodaca, P. \& Grad, H. (2002). Análisis dimensional de las opiniones de los alumnos universitarios sobre sus profesores. Revista de Investigación Educativa, 20(2), 385-409.

3. Arbeláez, R. (2004). Concepciones sobre una docencia universitaria de calidad. España: Universidad de Valencia.

4. Arrufat, M., Sánchez, V. \& Santiuste, E. (2010). El futuro docente ante las competencias en el uso de las tecnologías de la información y comunicación para enseñar. Tecnología educativa, 34 (1), 7 - 19.

5. Biggs, J. (2006). Calidad del aprendizaje universitario. Madrid: Narcea.

6. Bozu, Z., \& Herrera, P. (2009). El profesorado universitario en la sociedad del conocimiento: competencias profesionales docentes. Formación e Innovación Educativa Universitaria, 2(2), 221-231.

7. Bulgarín, R. (2008). Educación Superior en América Latina y el Proceso de Bolonia: Alcances y Desafíos. Ciencia UAT. Recuperado de http://goo.gl/4yfsRy

8. Bruner, J (2008). El proceso de Bolonia en el horizonte latinoamericano: límites y posibilidades. Revista de Educación, número extraordinario, $119-145$.

9. Brunner, J. (2010). Globalización de la educación superior: crítica de su figura ideológica. Iberoamericana de Educación Superior (RIES), 1 (2), $75-83$.

10. Cabalín, S. \& Navarro H. (2008). Conceptualización de los estudiantes sobre el buen profesor universitario en las carreras de salud de la Universidad de la Frontera - Chile. Internarional Journal o Morphology, 26(4), 887 - 892.

11. Cardona, A., Ayesta, M., Mijangos, J., Olaskoaga, J. (2009). Concepto y Determinantes de la Calidad de la Educación Superior. Un Sondeo de Opinión entre Profesores de Universidades Españolas. Archivos Analíticos de Politicas Educativas, 17(10).

12. Casero, M. (2010). ¿Cómo es un buen profesor universitario según el alumnado? Revista Española de Pedagogía, 68(264), 223- 242.

13. Castoriadis, C. (2007). La institución imaginaria de la Sociedad. Tomo II. Argentina: Tusquets.

14. Ceballos, D., Cantarero., D. \& Pascual, M. (2004). El tratado de Bolonia y la enseñanza superior: Una experiencia comparada de introducción de las Tics en ciencias económicas. Barcelona: Edutec.

15. Chacón, M. \& Pérez, L. (2001). Evaluación de la calidad universitaria: validez de contenido. Revista Psicothema, 2(13), 294-301.

16. CNA (2013). Acreditación de programas de pregrado en Colombia. Recuperado de www.cna.gov.co

17. Cordera, R. \& Santamaría, R. (2008). Internacionalización, autonomía y calidad de la educación superior: Elementos para la integración de América Latina y el Caribe. Revista Universidades, 37, 69 - 76.

18. Correira, S. \& Miranda, F. (2012). DUAQUAL: Calidad percibida por docentes y alumnos en la gestión Universitaria. Cuadernos de Gestión, 12(1), 107 - 122.

19. Demicheli, G. (2009). Calidad universitaria: actitudes y creencias de alumnos de respecto de un subgrupo de universidades de la región. Revista Calidad en la Educación. 31, 93-121. 
20. Díaz, J. (2008). Calidad, pertinencia y relevancia: relación con el resto del sistema y la sociedad; responsabilidad social de la educación superior. En. Gazzola, A., \& Pieres, S. (Comp.) Hacia una política regional de aseguramiento de la calidad en educación superior para América Latina y el Caribe. (pp. 16-55). Caracas: UNESCO-IESALC.

21. Díaz, E. (2010b). Bolonia y el arte de aprender. Revista de Derecho UNED, 7, 247-273.

22. Díaz, T., Maroto, I., Barcala, L., \& López C. (2012). Entornos personales de aprendizaje en el espacio europeo de educación superior. Revista Iberoamericana de Educación a distancia, 15(2), 123-142.

23. Díaz, A. (2013). El Plan Bolonia: retos y oportunidades en la gestión de prácticas para estudiantes. Capital Humano, 26(276), 26-28.

24. Esteban, R. \& Menjívar, S. (2011). Una mirada internacional a las competencias docentes Universitarias. Barcelona: Octaedro.

25. EURYDICE (2009): La educación superior en Europa 2009: progresos en el Proceso de Bolonia. Bruselas: EACEA.

26. Gacel, J. (2011). Impacto del proceso de Bolonia en la educación superior de América Latina. Revista de Universidad y SociedRad del Conocimiento, 8(2), 285-296.

27. Galán, P. (2009) Bases para una formación universitaria de calidad: ¿el EEES? La Cuestión Universitaria. Recuperado de http://www.lacuestionuniversitaria.upm.es/web/grafica/articulos/imgs_boletin_5/pdfs/LCU5-15.pdf?PHPSESSID=eme g9iigr2jngqolg3orndfkf2

28. García, V. (1991). La calidad de educación: un interrogante a las ciencias de la educación, a la política docentes y a la actividad escolar. En Consejo Superior de Investigaciones Científicas, Instituto de Pedagogía San José de Calasanz (Comp.). La calidad de la educación: experiencias científicas y condicionamientos individuales y sociales. (pp. 9 - 23). Madrid: Escuela Asturiana de estudios Hispánicos.

29. Gutiérrez, C. (2012). Equidad en la educación superior: una propuesta de financiación para Colombia. Revista Latinoamericana de Ciencias Sociales, Niñez y Juventud, 10(1), 397-413.

30. González, I. (2005). Dimensiones de evaluación de la calidad universitaria en el Espacio Europeo de educación superior. Revista Electrónica de Investigación Psicoeducativa, 10(3), 445 - 468.

31. González, L. (2005). El impacto del proceso de evaluación y acreditación en las universidades de América Latina. CINDA-IESALC/UNESCO.

32. Ibarra, I. \& Uribe, R. (2011). La competencia y las competencias docentes: Reflexiones sobre el concepto y la evaluación. Revista interuniversitaria de formación del profesorado. 35(14).

33. Iglesias, J., Saldívar, D., Bermúdez, V., \& Guzmán, A. (2005). Maltrato del estudiante de medicina. Percepción de 404 alumnos de cuarto, quinto y sexto años de la carrera. Revista Medicina Universitaria, 7(29), 191-202.

34. Jabif, L. (2007). La Docencia Universitaria Bajo un Enfoque de Competencias. Chile: Universidad Austral. 35. Lafuente, M. (2010). Calidad y experiencia docente en educación superior. Revista Historia de la Educación Latinoamericana. $15,177-196$.

36. Lago, D., López, E., Municio, P., Ospina, R., \& Vergara, G. (2013). La Calidad de la Educación Superior. ¿Un reto o una utopía? Bogotá: Ecoe. 


\section{CALIDAD DE LA EDUCACIÓN SUPERIOR: UN ANÁLISIS DE SUS PRINCIPALES DETERMINANTES}

37. Laudadío, J. (2012). Evaluación de estilos de enseñanza en la universidad: estudio preliminar de las propiedades psicométricas del cuestionario sobre la orientación docente del profesor universitario (CODPU). Revista de Psicología y Ciencias Afines, 29(1), 79-93.

38. Lavín, S. \& Del Solar, S. (2000). El proyecto educativo institucional como herramienta de transformación de la vida escolar. Chile: LOM.

39. Leiva, J. (2011). La participación del alumnado en la evaluación docente: Reflexiones para el debate en el contexto universitario. Revista de Formación e innovación educativa universitaria, 4(3), 170 - 176.

40. Lemaitre, M. (2004). Redes de agencias de aseguramiento de la calidad de la educación superior a nivel internacional y regional. Revista Iberoamericana de Educación. 35, 73 - 87.

41. Manzi, J. (2011). La evaluación docente en Chile. Chile: MIDE UC.

42. Mañú, J. (2011). Docentes competentes: por una educación de calidad. Madrid: Narcea.

43. Martínez, J. (2010). Universidad productora de productores: Entre biopolítica y subjetividad. Bogotá: Universidad de la Salle.

44. Mayor, C. (1997). El perfeccionamiento de los profesores universitarios principiantes a juicio de sus alumnos. Revista Interuniversitaria de Formación del Profesorado, 30, 127-149.

45. Marchesi, Á. (2007). Sobre el bienestar de los docentes: Competencias, emociones y valores. Madrid: Alianza.

46.Ministerio de Educación Nacional. (2010). Decreto 1295. www.mineducacion.gov.co/1621/article-229430.html.

47. Miranda, F., Chamorro, A. \& Rubio, S. (2007). Introducción a la gestión de la calidad. España: Delta.

48. Montero, M (2010). El Proceso de Bolonia y las nuevas competencias. Revista Tejuelo, 9, 19 - 37.

49. Municio, P. (2000). La evaluación de la calidad en educación. Madrid: Narcea-Consudec.

50. Murcia, N. (2009). Vida universitaria e imaginarios: posibilidad en definición de políticas sobre educación. Revista Latinoamericana de Ciencias Sociales Niñez y Juventud, 7(1), 235-266.

51. Pales, J., Nolla, M., Oriol, A. \& Gual, A. (2010). Proceso de Bolonia: educación orientada a competencias. Revista Educación médica, 13(3), 127-135.

52. Ponce, E. (2012). La educación superior en Chile y el rol del mercado: ¿culpable o inocente? Revista Chilena de Ingeniería - INGENIARE, 20(1), 126-135.

53. Porter, L. (2005). La universidad de papel: Ensayos sobre la educación superior en México. México: Instituto de Investigación y Desarrollo Educativo de la Universidad Autónoma de Baja California

54. Rodríguez, E. (2006). La reforma de la educación superior en América Latina: Algunas reflexiones sobre la calidad de la enseñanza universitaria, formación del profesorado y las reformas institucionales actuales, a partir del análisis del debate en la universidad Uruguaya. Revista Unipluriversidad, 6 (2), 85 - 98.

55. Rodríguez, A., Ayesta, M., Mijangos, J. \& Olaskoaga J. (2009). Concepto y Determinantes de la Calidad de la Educación Superior. Un Sondeo de opinión entre profesores de universidades españolas, Archivos Analiticos de Políticas Educativas, 17(10), 1- 22. 
56. Rodríguez, A. \& Vieira, M. (2009). La formación en competencias en la universidad: Un estudio empírico sobre su tipología. Revista de Investigación Educativa, 27(1), 27 - 47.

57. Ruiz, I., Cano, C., \& Montes, I. (2008). Financiamiento y demanda de educación superior en Colombia (periodo 1990 2005). Revista calidad en la educación, 29, 111-136.

58. Rueda, M. (2009). Evaluación del desempeño docente: consideraciones desde el enfoque por competencias. Revista electrónica de investigación educativa, 11(2), 1 -16.

59. Salazar, J. \& Leihy, S. (2013). El Manual Invisible: Tres décadas de políticas de educación superior en Chile (19802010). Archivos Analíticos de Politicas Educativas, 21(34), 2 - 37.

60. Scharager, J. \& Aravena, M. (2010). Impacto de las políticas de aseguramiento de la calidad en educación superior: un estudio exploratorio. Revista Calidad en la Educación, 32, 16-42.

61. Soto, V. (2002). Políticas, acciones curriculares y reforma de la educación en Chile. Estudios y experiencias en educación, $1(1), 57-74$.

62. Tejedor, F. \& García, A. (2010). Evaluación del desempeño docente. Revista española de pedagogía, 68(247), 439-459.

63. Tirado, M. (2009). Docencia universitaria y competencias didácticas. Revista Perfiles Educativos, 31 (125), $76-87$.

64. Torres, E. \& Araya, L. (2010). Construcción de una escala para medir la calidad del servicio de las universidades: Una Aplicación al Contexto Chileno. Revista de Ciencias Sociales, 16(1), 54 - 67.

65. Torres, D. \& Zapata, G. (2012). Impacto de procesos de aseguramiento de la calidad sobre las instituciones de educación superior: un estudio en siete países. En: Lemaitre, M., \& Zenteno, M. (Coord.). Aseguramiento de la calidad en Iberoamérica: Educación superior informe 2012 (pp. 167 - 145). Chile: CINDA.

66. Zabalza, M. \& Zabalza, M. (2010). Planificación de la docencia en la universidad: Elaboración de las guías docentes de las materias. Madrid: Narcea. 
